\title{
Perspective
}

PERSPECTIVE Actualité en histoire de l'art

2 | 2020

Danser

\section{Sculptures vivantes : réception et recréation des danses indiennes en Occident (1900-1939)}

Living Sculptures: The Reception and Recreation of Indian Dances in the West (1900-1939)

Lebende Skulpturen: Rezeption und Neuschöpfung indischer Tänze in der westlichen Welt (1900-1939)

Sculture viventi: ricezione e ricreazione delle danze indiane in Occidente (1900-1939)

Esculturas vivas: recepción y recreación de las danzas indias en Occidente (1900-1939)

\section{Irene López Arnaiz}

Traducteur : Sophie Zambrano

\section{(2penEdition} Journals

\section{Édition électronique}

URL : https://journals.openedition.org/perspective/21010

DOI : 10.4000/perspective. 21010

ISSN : 2269-7721

\section{Éditeur}

Institut national d'histoire de l'art

\section{Édition imprimée}

Date de publication : 30 décembre 2020

Pagination : 179-194

ISBN : 978-2-917902-90-5

ISSN : $1777-7852$

\section{Référence électronique}

Irene López Arnaiz, «Sculptures vivantes : réception et recréation des danses indiennes en Occident (1900-1939) », Perspective [En ligne], 2 | 2020, mis en ligne le 30 juin 2021, consulté le 30 juillet 2022. URL : http://journals.openedition.org/perspective/21010; DOI : https://doi.org/10.4000/perspective. 21010 


\title{
Sculptures vivantes : réception et recréation des danses indiennes en Occident (1900-1939)
}

\author{
Irene López Arnaiz
}

La sensualité a pris, notamment en Orient, la forme singulière d'une sorte de mysticisme charnel, dont l'on retrouve des exemples dans les rites grecs et romains et dans certaines de nos étranges fêtes médiévales ${ }^{1}$.

En 1928, Anton Giulio Bragaglia publie l'ouvrage Scultura Vivente, dans lequel il décrit le caractère naturel et instinctif de la danse à partir de l'évocation du rapport de celle-ci avec les arts visuels. Le livre aborde quelques-uns des thèmes fondamentaux autour desquels s'articulent, aux $\mathrm{XIX}^{\mathrm{e}}$ et $\mathrm{XX}^{\mathrm{e}}$ siècles, la réception et la recréation des danses indiennes en Occident, en lien étroit avec les processus de modernisation de la danse qui caractérisent cette période. Les relations entre cette discipline et le champ ésotérique et sacré sont mises en évidence à travers l'évocation de la présence primordiale de cet art dans les civilisations antiques, un thème central pour les prémices de la danse moderne. En consacrant un chapitre entier à Nyota Inyoka, Bragaglia témoigne de l'intérêt que suscitaient les danses indiennes et leur dimension sacrée dans l'Europe des premières décennies du $\mathrm{XX}^{\mathrm{e}}$ siècle. En outre, les interrelations profondes entre disciplines artistiques qui caractérisent l'avant-garde ressortent nettement de l'ensemble de l'ouvrage comme du parcours artistique et intellectuel de l'auteur lui-même. Photographe et cinéaste appartenant au courant futuriste italien, il éprouva un vif intérêt pour la danse et le théâtre et créa à Rome la Casa d'Arte Bragaglia et le Teatro Sperimentale degli Indipendenti, où les artistes et les intellectuels de l'époque eurent l'occasion de se rencontrer.

La curiosité croissante envers les cultures non européennes qu'engendra l'impérialisme allait favoriser la réception de formes scéniques exotiques dans un certain nombre de métropoles occidentales, parmi lesquelles Paris fait figure de centre artistique incontournable dans le passage du XIX ${ }^{e}$ au XX ${ }^{e}$ siècles ${ }^{2}$. Un Orient luxueux, sacré et érotique, aux contours diffus, qui s'étendait du sud des Pyrénées à l'ensemble de l'Asie, excitait l'intérêt du public. Dans ce contexte, la réception des danses de l'Inde et du Sud-Est asiatique mettait davantage l'accent sur leurs affinités thématiques et formelles que sur leurs particularités et origines diverses.

Nous traiterons dans les pages qui suivent, premièrement, la question de la réception en France des danses de l'Asie du Sud et du Sud-Est, afin de montrer leur présence marquante dans le paysage artistique et culturel des premières décennies du $\mathrm{XX}^{\mathrm{e}}$ siècle. 
Bien que Paris occupe une place centrale, le circuit des scènes de l'époque comprend plusieurs villes européennes et nord-américaines, mais aussi asiatiques et même latinoaméricaines, faisant de la danse un vecteur très efficace des échanges artistiques et culturels de la modernité. Deuxièmement, la présence démontrée des danses indiennes dans ces contextes et l'admiration que leur vouèrent des artistes et des intellectuels de l'époque nous amèneront à affirmer l'influence majeure de ces danses dans les cercles de la modernité de la première moitié du XX $\mathrm{XX}^{\mathrm{e}}$ siècle. Aussi nous attacherons-nous, dans la présente contribution, à étudier les créations scéniques d'inspiration indienne apparues sur la scène occidentale

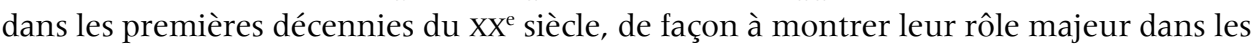
processus de rénovation de la danse à la même période ${ }^{3}$. L'approche interdisciplinaire adoptée dans ces pages est particulièrement liée au recours de beaucoup de ces créatrices aux arts visuels de l'Asie du Sud et du Sud-Est en tant qu'outil de légitimation et source d'inspiration de leurs propositions artistiques. Cet aspect, qui contribua longtemps à l'obscurcissement de leur travail créatif, doit cependant être mis en regard des propositions d'autres danseuses plus connues comme Isadora Duncan, considérée par l'historiographie comme une pionnière majeure de la danse moderne.

Cet essai vise en fin de compte à réhabiliter le travail de nombreuses danseuses aux répertoires inspirés des danses indiennes qui furent délibérément oubliées, ou à tout le moins dédaignées par l'historiographie plus généraliste. Par ailleurs, l'étude du processus créatif de ces artistes fait apparaître d'importantes résonances avec bon nombre d'enjeux de la modernité du début du $\mathrm{XX}^{\mathrm{e}}$ siècle, laquelle fut fortement marquée par les interactions entre différents langages artistiques et par leurs références à des traditions ancestrales reliées au sacré.

\section{Un tour du monde imaginaire : arrêt sur les danses " indiennes "}

Dans son chapitre consacré aux danses orientales, André Levinson relevait l'image fragmentaire que se faisaient de ce territoire diffus les Occidentaux, évoquant les « impressions aiguës, mais isolées " nées de ces formes scéniques, qui lui permettaient cependant de faire le tour du monde en imagination ${ }^{4}$. Levinson affirme : "Nous n'avons vu l'Asie qu'exhibée sur les tréteaux européens "; et il se questionne : "Ce voyage immobile suffit-il pour que nous essayions de conclure ? Peut-être, se répond-il à lui-même, car avoir vu danser un indigène, c'est presque avoir visité son pays. " Ces propos du critique français, outre qu'ils confirment la présence marquante des danses asiatiques sur la scène française, semblent tirer leur origine de l'assimilation des cultures exotiques qui s'était produite en Europe depuis le siècle précédent. En 1843, Théophile Gautier exprima une impression similaire, bien qu'inversée, à propos de la danse espagnole. Déçu par le boléro qu'il vit danser à Vitoria, l'écrivain déclara ainsi « les danses espagnoles n'existent qu'à Paris, comme les coquillages, qu'on ne trouve que chez les marchandes de curiosités, et jamais sur le bord de la mer ", avant d'attribuer à Fanny Elssler l'invention de la cachucha ${ }^{5}$. Si, plus tard, Gautier allait apprécier les danses de l'Andalousie, ces deux commentaires mettent en exergue la capacité des scènes internationales d'accueillir des danses étrangères, de les adapter et même de les recréer selon leurs traditions propres, assouvissant une soif d'exotisme présente depuis le romantisme tout en véhiculant une image stéréotypée de l'autre, projetée sur ces corps en mouvement. Cet aspect est particulièrement important si l'on tient compte de la double nature de ce qu'Anne Décoret-Ahiha définit comme le genre des danses hindoues, objet de la présente étude, développé essentiellement par des femmes. Les danseuses hindoues se réclamaient d'origines orientales plus ou moins 
romancées, entourant leur biographie de mystère de façon à souligner l'authenticité de leurs danses et à incarner le topique exotico-sacré de la danseuse indienne, né de l'imaginaire européen et recherché encore de nos jours. Or, la plupart de ces chorégraphies furent créées en Occident et pour l'Occident, à partir de sources visuelles et textuelles toujours plus présentes sur le continent européen. Cette étude plus ou moins rigoureuse des sources allait de pair avec un remarquable travail créatif, à une époque où la danse occidentale avait amorcé son renouveau à travers l'expérimentation de divers langages alternatifs. Toutefois, l'inclusion de ces danses dans l'ensemble abondant et disparate des répertoires orientalisés et " exotisés " de l'époque, de même que le débat qui entoura dès le début la question de leur authenticité ou de leur falsification, contribuèrent fortement à ce que l'historiographie du XXe siècle sous-estime la valeur et l'importance de ces compositions.

L'évocation récurrente du récit de Jules Verne servait déjà, au XIX ${ }^{e}$ siècle, à invoquer la présence de cultures multiples en Occident. C'est ce qui ressort du témoignage d'Henry Fouquier au sujet de l'Exposition universelle de Paris de 1889 : "Tout d'abord, avec un peu d'imagination et quelque lecture, l'Exposition était pour bien des gens un véritable voyage autour du monde, plus rapide, moins fatigant et moins coûteux que le Voyage en quatre-vingts jours raconté par M. Verne ${ }^{6}$. " Les expositions coloniales et universelles françaises de la fin du XIX ${ }^{e}$ siècle et du premier tiers du XX ${ }^{e}$ offraient un espace de rencontre avec des cultures non européennes par l'intermédiaire d'objets, de manifestations artistiques et, surtout, des arts de la scène de différentes contrées. Outre les danses d'Asie du Sud et du Sud-Est, sur lesquelles nous reviendrons brièvement un peu plus loin, il faudrait encore mentionner la danse du ventre de la rue du Caire à l'Exposition de 1889, ou celle de la Belle Fatma, présentée au Grand Théâtre lors de la même manifestation, juste avant que « les Gitanes de Grenade y vinssent faire leur bruyante et triomphante apparition ${ }^{7}$ » La prestation de Sada Yacco au Théâtre de Loïe Fuller à l'Exposition universelle de 1900 éveilla l'intérêt d'artistes tels que Rodin ou Picasso, et Rudolf von Laban fit entrer la danse moderne à l'Exposition des Arts décoratifs de 1925 à Paris .

Pour ce qui est des danses de l'Asie du Sud et du Sud-Est, en 1838, un groupe de cinq devadàsī (danseuses sacrées) originaires d'un temple de Pondichéry entamèrent une tournée en Europe, surprenant un public accoutumé à la bayadère représentée par le ballet ${ }^{9}$. Par la suite, et jusqu'à l'entre-deux-guerres, plusieurs groupes de danseuses indiennes allaient se succéder dans les expositions universelles et coloniales, les zoos humains et les music-halls, celles du Sud-Est asiatique étant particulièrement prisées ${ }^{10}$. Les Javanaises de l'Exposition universelle de Paris, en 1889, firent vive impression sur Auguste Rodin, Henri de Toulouse-Lautrec, Paul Gauguin, Camille Pissarro ou John Singer Sargent ; Rodin immortalisa le Ballet royal du Cambodge à l'Exposition coloniale de Marseille en 1906, tandis qu'Antonin Artaud fut ébloui par les Balinaises de l'Exposition coloniale de Vincennes en $1931^{11}$.

Au changement de siècle, ces danses exotiques jugées " authentiques " cohabitèrent avec les chorégraphies inspirées de l'Asie du Sud composées, en majorité, par les danseuses hindoues, dont le qualificatif, en usage à l'époque, mettait en exergue le caractère sacré des traditions scéniques invoquées. Le genre des danses hindoues vit le jour au musée Guimet en 1905 avec les « danses brahmaniques » interprétées par Mata Hari pour illustrer une conférence sur les danses védiques ${ }^{12}$. En 1900, Cléo de Mérode avait composé pour le théâtre des Capucines une pièce javanaise, prélude à ses créations cambodgiennes pour l'Exposition universelle qui se tint la même année ${ }^{13}$ (fig. 1). Ces compositions annonçaient les spectacles d'inspiration indienne qui seraient présentés dans des espaces analogues, contribuant à l'émergence de la danse moderne comme à la diffusion des traditions scéniques indiennes. 
Lors de l'Exposition des Arts décoratifs de 1925, Vanah Yami exécuta des danses javanaises. À cette même occasion, Cosika Vrandja dansa des pièces khmères auxquelles s'ajoutèrent les Danses de la cour de Cachemire qu'elle interpréta aux côtés d'Uday Shankar ${ }^{14}$. La présence du danseur indien à l'Exposition mérite d'être soulignée, à plus forte raison si l'on considère qu'elle date des débuts d'une carrière initiée quelques années plus tôt auprès d'Anna Pavlova, puis avec le soutien d'Alice Boner ${ }^{15}$. Bien qu'il ait intégré la scène indienne à partir de 1940, les premières compositions d'Uday Shankar étaient destinées au public occidental ; Joan L. Erdman qualifia ces pièces de « traductions » des danses indiennes, qui offraient un caractère authentique tout en restant accessibles au public européen ${ }^{16}$. Il convient de garder à l'esprit que ces compositions entrent en scène en plein entre-deux-guerres, à une époque où ces danses font fureur en Europe et aux États-Unis et au moment où le danseur, encouragé par deux femmes incontournables de la scène chorégraphique et artistique de l'époque, redécouvre sa propre tradition ${ }^{17}$ (fig. 2). Si, aux " traductions " d'Uday Shankar, l'on ajoute les adaptations des danses javanaises présentées à l'Exposition universelle de 1889, on devine l'impact qu'a pu avoir la réception occidentale sur la transformation de ces traditions scéniques à l'aube de la modernité. Claudia Palazzolo signale que le vocabulaire chorégraphique ou la trame des danses javanaises furent adaptés ou réduits et que les musiciens qui accompagnaient les danseuses étaient originaires d'autres

1. "Cléo de Mérode dans les danses cambodgiennes", photographie issue de l'album Reutlinger (1875-1917), vol. 6, p. 13, Paris, Bibliothèque nationale de France, département Estampes et Photographie.

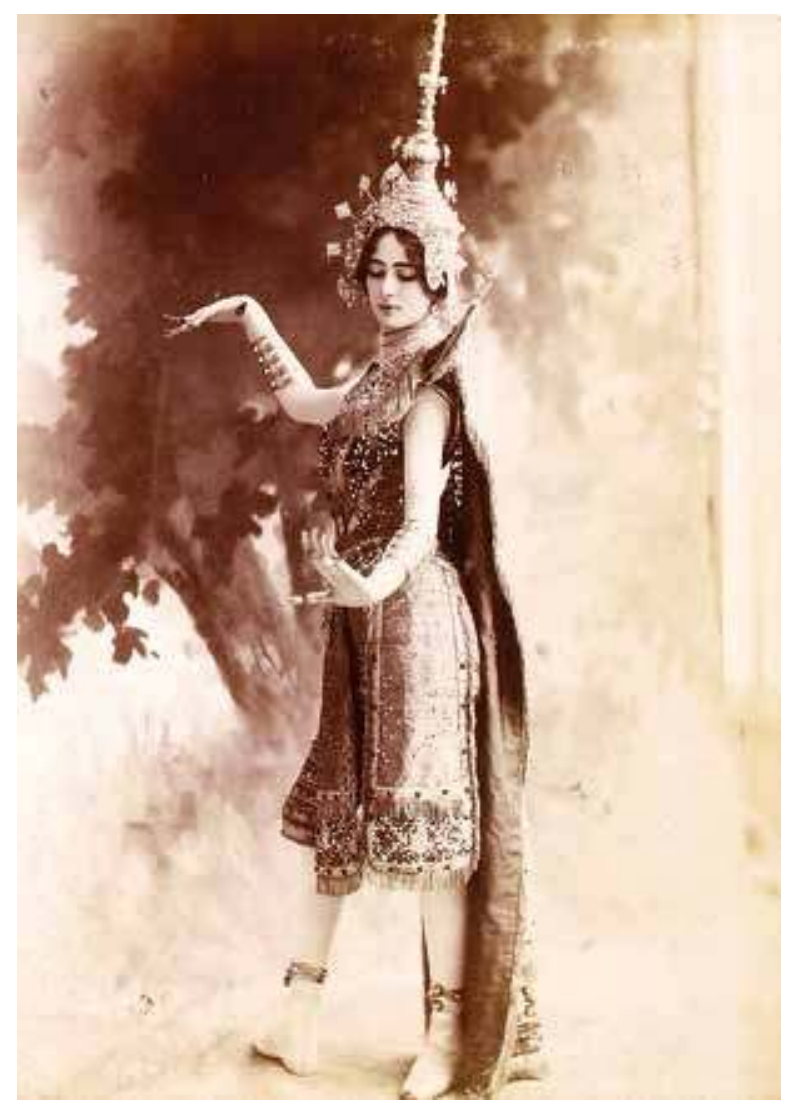
régions, aux répertoires distincts ${ }^{18}$. Cela ne fait que confirmer les liens étroits qui s'établirent, dans les premières décennies $\mathrm{du} \mathrm{XX}^{\mathrm{e}}$ siècle, entre la scène européenne et celles de l'Asie du Sud et du Sud-Est, et qui contribuèrent à estomper les frontières à la fois entre modernité et tradition et entre des contextes géographiques très éloignés. On commença à remettre en question l'opposition des catégories authentique / traditionnel et faux / moderne qui prévalait à l'époque et dont s'est imprégnée une partie de l'historiographie - remise en question qu'il faut reconduire aujourd'hui.

La présence d'Uday Shankar à l'Exposition de 1925 allait avoir un impact majeur sur deux de ses contemporaines qui participaient elles aussi à la manifestation, à savoir les danseuses Jeanne Ronsay et Nyota Inyoka ${ }^{19}$. La première l'invita à enseigner dans son école, où Shankar fit la connaissance de Michelle Damour et de Simone Barbier qui adoptera plus tard le nom de Simkie ; l'une et l'autre ont fait partie de sa compagnie ${ }^{20}$.

À l'occasion de l'Exposition coloniale de Vincennes de 1931 et de l'Internationale des Arts et Techniques de 1937, on vit revenir sur la scène française des danses indiennes différentes, interprétées par des artistes dont 
la contribution à ce que Ramsay Burt et Michael Huxley nomment la « dimension globale du modernisme $^{21} »$ fut essentielle ${ }^{22}$ (on citera notamment les danses indiennes ou javanaises présentées par Nyota Inyoka, Djemil Anik, La Meri et Uday Shankar ${ }^{23}$ ). Burt et Huxley soulignent par cette expression la capacité de la danse des premières décennies du $\mathrm{XX}^{\mathrm{e}}$ siècle à établir des liens transnationaux plus facilement que d'autres langages artistiques, ce dont témoignent les nombreuses tournées effectuées par les danseurs de l'époque dans diverses régions du monde. En ce sens, notre étude tend à confirmer l'idée d'un effacement des contours de la modernité au profit de modernités plurielles et décentralisées, nécessitant la prise en compte d'une pluralité de perspectives et de références culturelles. Nyota Inyoka, Djemil Anik ou encore Uday Shankar, qui participèrent à des manifestations impérialistes de cette période, comme les expositions citées, en sont justement l'illustration parfaite. Tandis que les premières grandirent en France mais n'eurent de cesse de revendiquer leurs origines métisses, Uday Shankar, né en Inde britannique, débuta sa carrière en tant que danseur parfaitement intégré au circuit des scènes occidentales et destina ses premières créa-

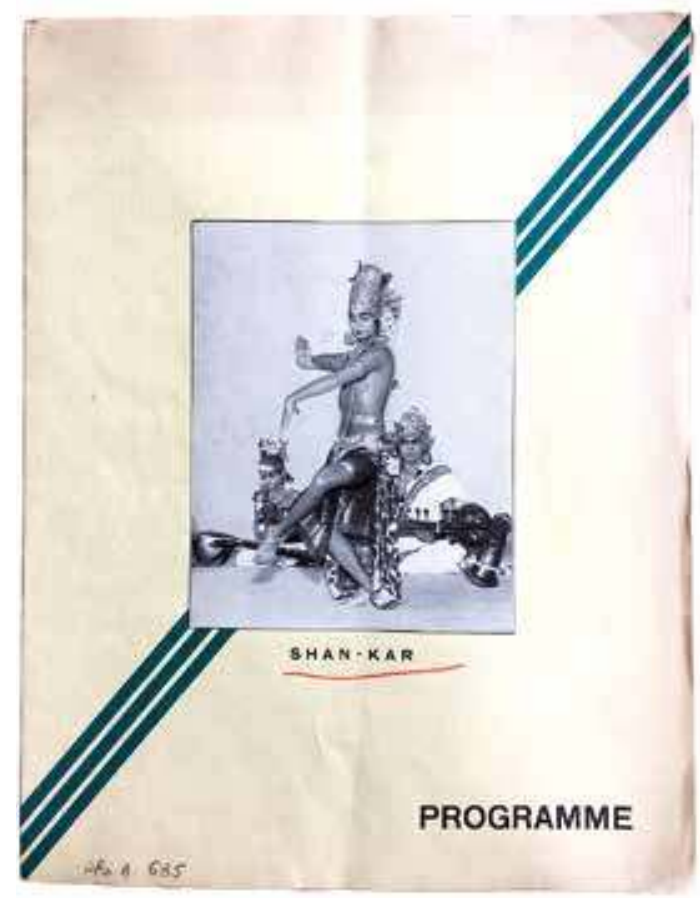

2. Uday Shankar, programme «Uday Shan-kar et Simkie avec Orchestre hindou », Théâtre des Champs-Élysées, 3 mars 1931, Paris, Bibliothèque nationale de France, bibliothèquemusée de l'Opéra. tions au public européen. Si l'on considère cela, mais aussi les questions raciales autour desquelles s'articule le travail des corps constitutif de l'art de la danse et la conjonction d'influences occidentales et asiatiques présente dans ces créations chorégraphiques, elles-mêmes reliées à des questions identitaires, le recours à la dimension globale de la modernité revendiquée pour la danse par Burt et Huxley s'impose comme une nécessité évidente. C'est l'une des raisons pour lesquelles, jusqu'à récemment, il était malaisé d'insérer le travail des danseuses hindoues, à mi-chemin entre la scène européenne et l'imaginaire exotique, dans les circuits de la modernité, l'œuvre d'Uday Shankar se trouvant quant à elle reléguée au domaine de la danse moderne spécifiquement indienne. En ce sens, les contacts établis entre l'Europe et le reste du monde dans le contexte des politiques impériales, clairement sous-tendues par des dynamiques de pouvoir, doivent nous inciter à une relecture en termes globaux de l'art moderne de l'époque, comme le proposent des spécialistes récents du domaine des études postcoloniales et décoloniales ${ }^{24}$.

\section{Le retour aux sources : une archéologie recréée de la danse}

" Il pourrait bien se faire que la danse n'ait pas attendu la musique, ni même la parole pour se manifester. Expression irrésistible de la joie de vivre, elle a dû être la première tentative de l'homme pour organiser ses mouvements ", écrivait Francis de Miomandre ${ }^{25}$. La revendication de la danse en tant qu'acte artistique originel de l'être humain est une constante des premières décennies du Xx $x^{e}$ siècle. Elle s'inscrivait dans un processus 


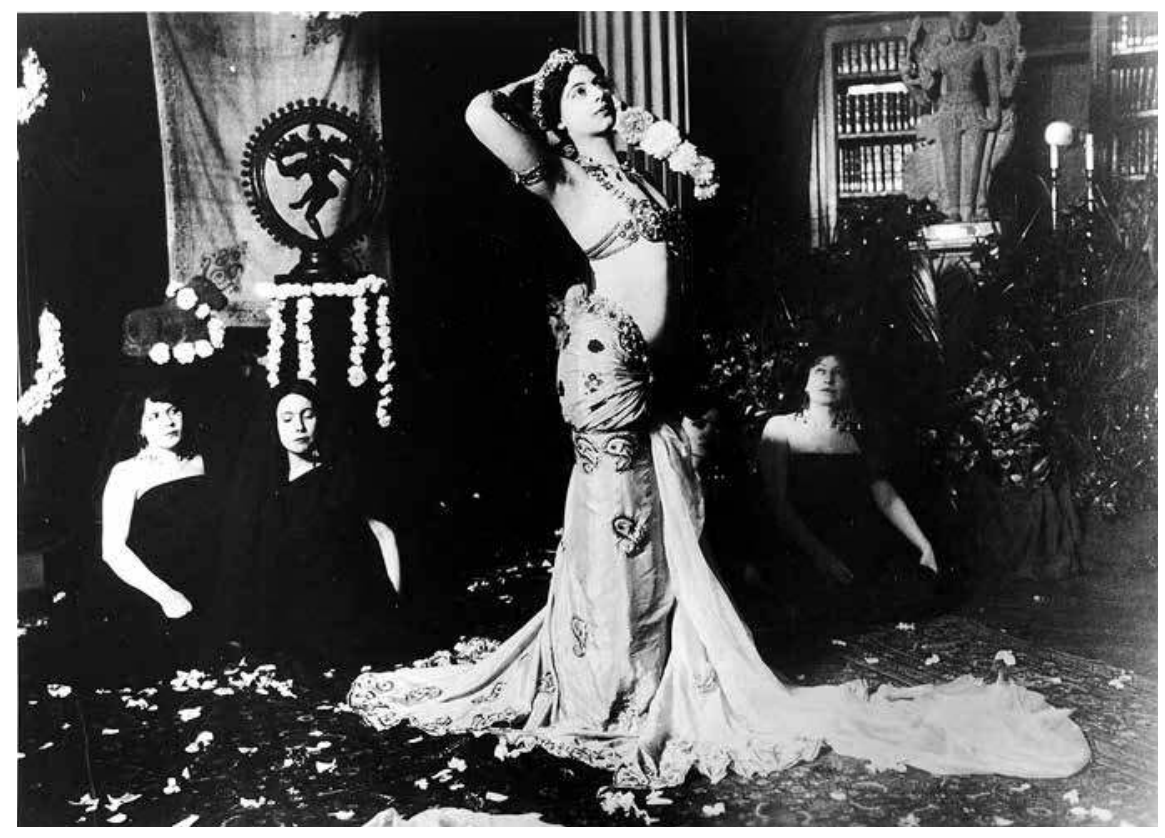

3. Mata Hari exécutant des danses brahmaniques dans la bibliothèque du musée Guimet à Paris, 1905, Paris, Musée national des arts asiatiquesGuimet. de légitimation de cette discipline, considérée comme un art élevé et pas uniquement comme un divertissement frivole. Cette stratégie fit coïncider le regard porté sur l'antiquité grecque avec les répertoires éclectiques orientaux, eux aussi tributaires d'anciennes traditions liant étroitement la danse aux pratiques religieuse ${ }^{26}$.

Bien qu'on présentât ses propositions en termes de "reconstitutions ", ce retour vers le passé participait de toute évidence du renouveau de l'art de la danse, en interaction, bien souvent, avec d'autres disciplines. La volonté d'émanciper le corps des artifices du ballet, dans la recherche d'une danse naturelle, trouvait en Grèce la quintessence de cette appréhension du mouvement. Isadora Duncan fait figure de référence incontestable dans ce processus de libération fondé sur l'étude de l'art de l'antiquité grecque et de la Renaissance dans des musées de Londres et de Paris, que l'on pourrait qualifier de travail archéologique tout autant que créatif. Ce retour au classicisme occidental est contemporain des propositions des danseuses hindoues qui, à la même époque, puisent leur inspiration dans les arts visuels des cultures qu'elles ambitionnent d'incarner. Il faut mentionner aussi la publication en Europe ou la traduction, en anglais ou en français, d'une profusion d'études de plus en plus rigoureuses sur l'art de l'Inde et du Sud-Est asiatique dans les dernières décennies du XIX ${ }^{e}$ siècle et les premières du XX ${ }^{e}$. Aux études de figures majeures comme Ernest Binfield Havell, Sylvain Lévi, Ananda K. Coomaraswamy ou George Groslier ${ }^{27}$, s'ajoute un afflux croissant de pièces en provenance d'Asie du Sud et du Sud-Est exhibées lors des expositions universelles et dans les musées européens, qui témoignent de l'essor des missions archéologiques dans ces régions ${ }^{28}$. On peut lire à ce propos, dans un document inédit de la danseuse Nyota Inyoka destiné à faire connaître ses créations : "Les danses de Nyota Inyoka s'intègrent pour une large part dans l'archéologie de l'Orient, sa mythologie, sa science sacrée, sa philosophie. " Ce document met également en avant la valeur de l'étude de l'art, de la pensée et des religions de l'Inde et de l'Égypte dans le processus créatif de l'artiste ${ }^{29}$. 

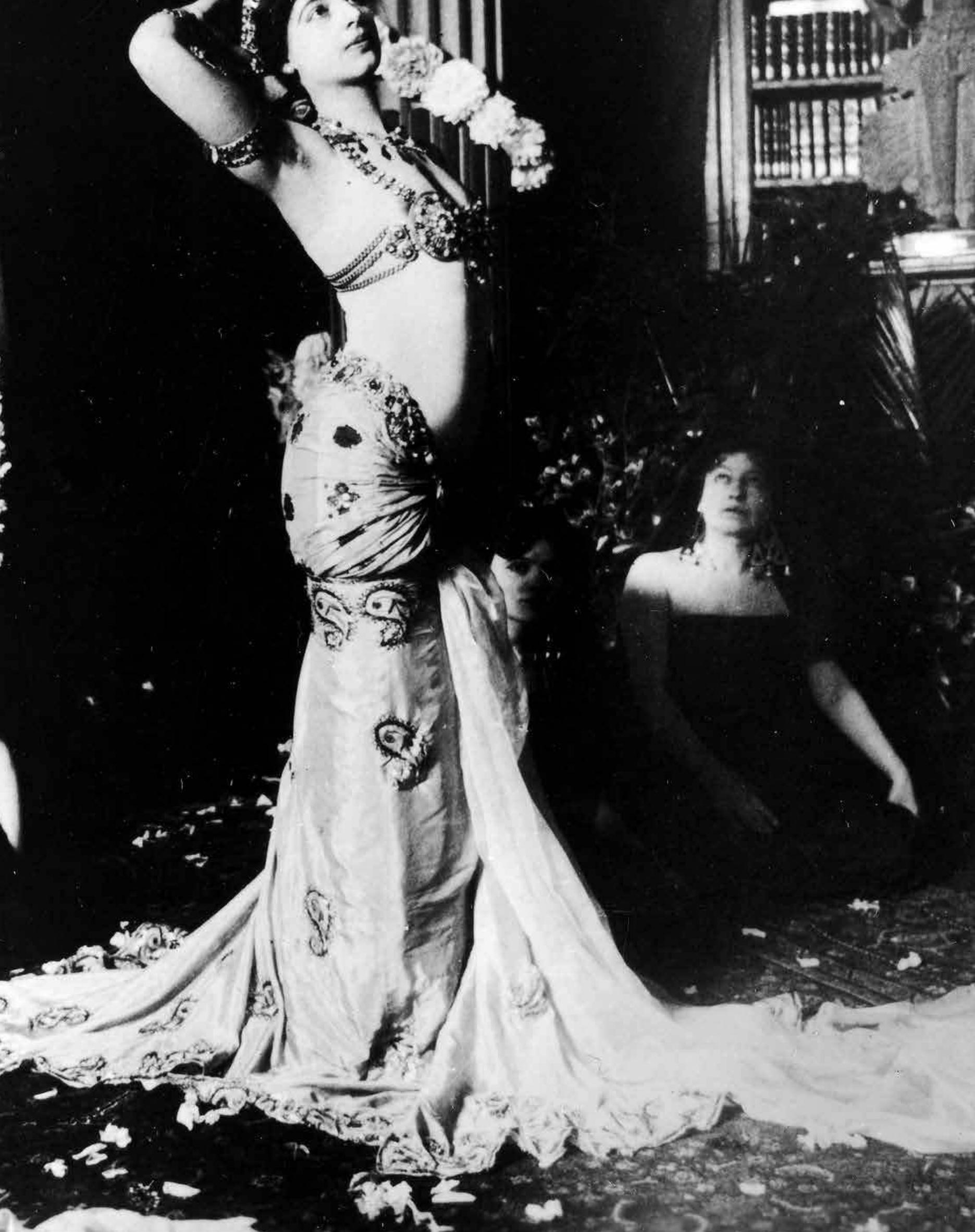
Gabriele Brandstetter propose une analyse intéressante du lien entre l'origine de la danse moderne et l'Antiquité grecque, en établissant un parallèle avec les formules du pathos théorisées par Aby Warbug ${ }^{30}$. On retrouve aussi cette symbiose entre le musée et la danse moderne dans les propositions indiennes du premier tiers du XX $\mathrm{X}^{\mathrm{e}}$ siècle. Depuis Cléo de Mérode - qui affirmait s'être inspirée de gravures et de sculptures pour ses danses cambodgiennes - jusqu'à Nyota Inyoka, les arts visuels s'imposent comme la référence absolue dans la composition des danses hindoues ${ }^{31}$. La prestation de Mata Hari à la bibliothèque du musée Guimet illustre bien ce dialogue entre la sculpture et la danse, qui permettait en outre de transformer la scène en un temple hindou, sacralisé par un Națarāja ${ }^{32}$ (fig. 3). L'espace muséal, aussi bien que les œuvres d'art, conféraient une légitimité au spectacle de la Hollandaise, dont le caractère lascif renvoyait en même temps à l'imaginaire occidental de la danseuse indienne, qui associait immanquablement l'érotisme et le sacré33.

Ruth Saint Denis décrivit sa méthode de composition à partir d'images de devadāsì consultées à New York, à l'Astor Library, ainsi que de sculptures contemplées au Metropolitan Museum of Art et dans d'autres musées européens ${ }^{34}$. Pour citer Tiziana Leucci ${ }^{35}$, l'Inde fut pour cette artiste un moyen de "légitimation de sa propre quête religieuse $^{36}$ ", à laquelle l'avaient amenée - entre autres - l'art et la philosophie de l'Inde ${ }^{37}$. Sa pièce Radha (1906), qualifiée de " danse mystique ", révèle ses recherches à partir de différentes sources visuelles et son étude des textes hindous et bouddhistes ${ }^{38}$ (fig. 4). Cette quête d'une danse transcendantale qui la relierait au passé de l'Inde

4. Ruth Saint Denis dans Radha, 1906, The New York Public Library, Jerome Robbins Dance Division. conduisit Crawford Flitch à considérer la danseuse, plutôt que comme une artiste créatrice, comme une simple imitatrice ou traductrice trouvant ses références dans l'art

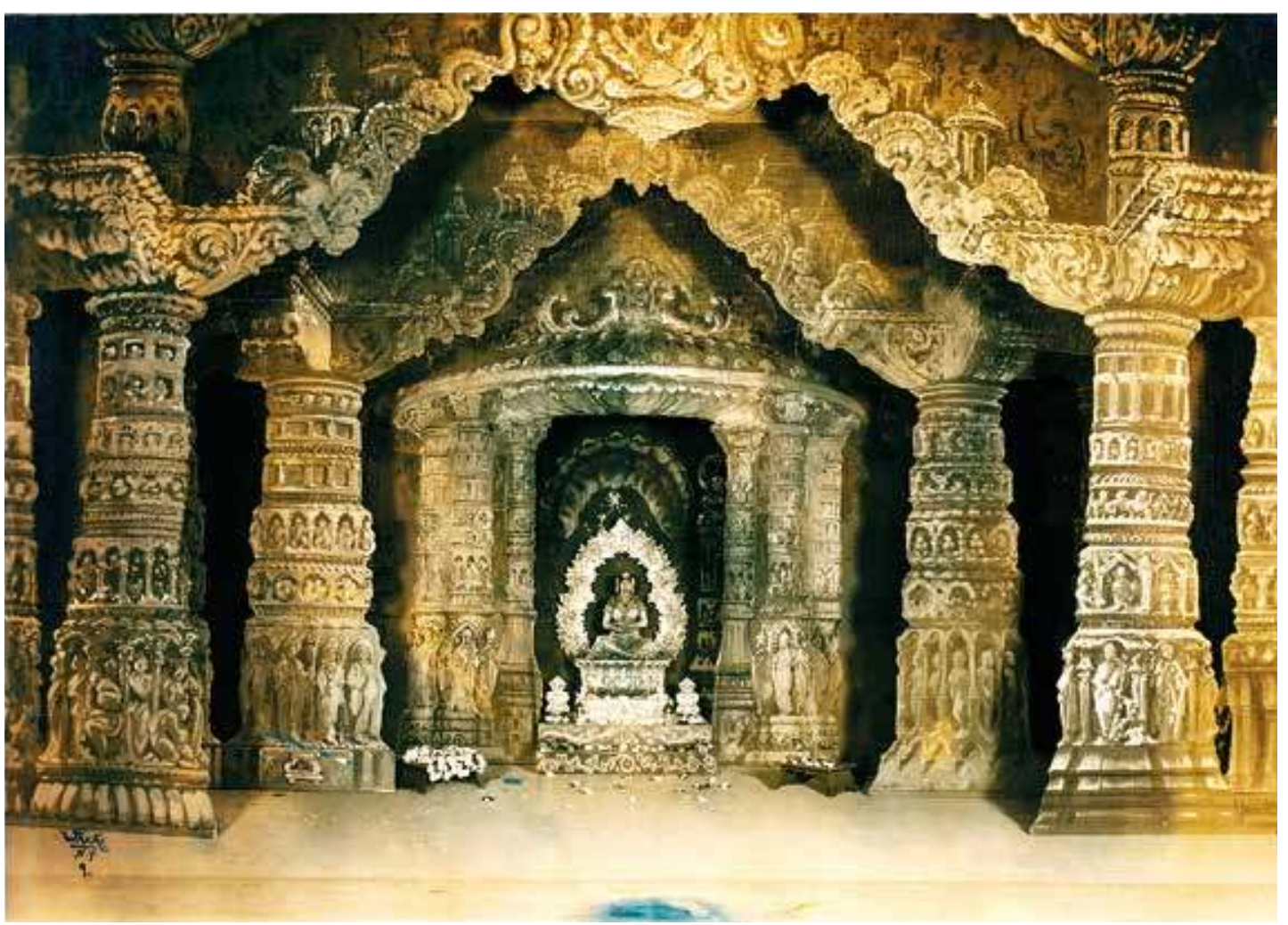




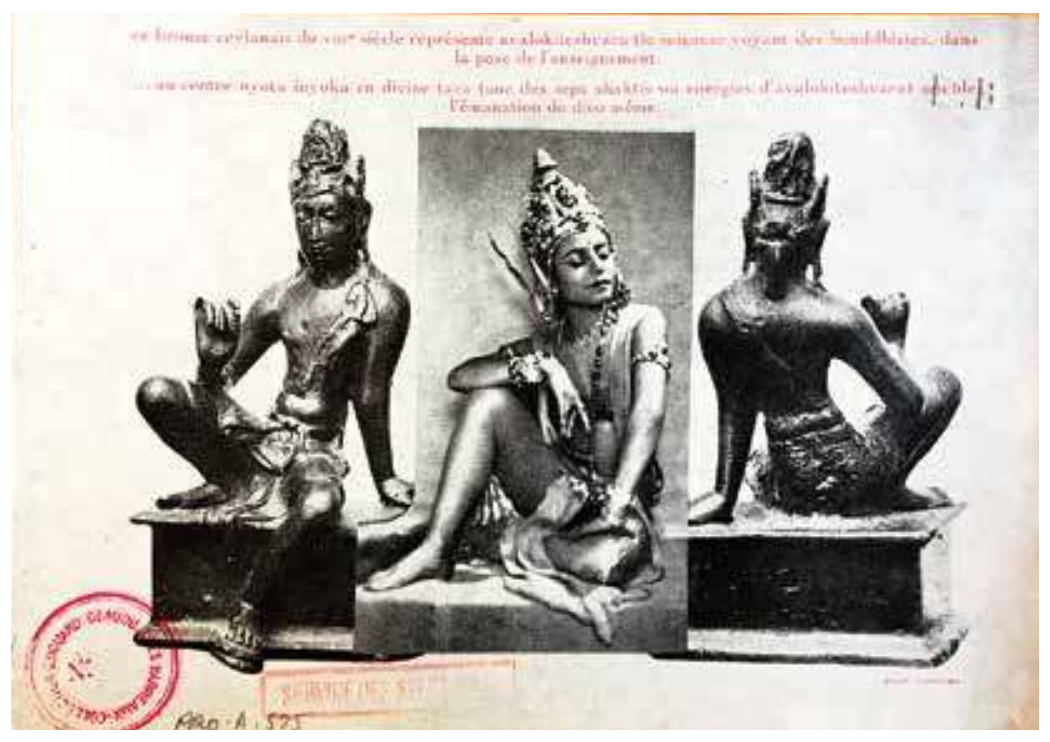

et la religion indiennes ${ }^{39}$. L'auteur britannique attirait l'attention sur la dichotomie récurrente, dans les danses hindoues, entre l'évocation de 5. Nyota Inyoka au bénéfice de l'œuvre "Le colis tricolore " (carton d'invitation), Salle Gaveau, Paris, 25 avril s.a., Paris, Bibliothèque nationale traditions ancestrales et la création de propode France, bibliothèque-musée de l'Opéra.

sitions modernes.

Carmen Tórtola Valencia, dont le répertoire éclectique comprenait des danses espagnoles, arabes, grecques, indiennes et incas, se livrait à un véritable travail de documentation à partir des images qu'elle collectionnait et de ses visites au musée du Louvre, au British Museum et dans des musées espagnols ${ }^{40}$. Comme beaucoup de danseuses de son époque, l'Espagnole se servit des œuvres d'art pour remonter aux origines mêmes de la danse ${ }^{41}$.

André Levinson évoquait « la recherche des indices et vestiges du mouvement saltatoire " de Nyota Inyoka, qui puisait à son tour son inspiration dans des "idoles de bronze et de pierre avidement scrutées ». L'auteur comparait sa méthode à celle de Djemil Anik, visant à " restitue[r] les rites de l'Ancient Orient ${ }^{42}$ ». Nombreux sont les témoignages qui décrivent Nyota Inyoka copiant des pièces du musée Guimet ou de la bibliothèque d'art et d'archéologie Jacques Doucet ${ }^{43}$. Parallèlement à son étude toujours plus approfondie de la culture indienne, Nyota Inyoka se servit des arts visuels comme d'un outil documentaire et créatif de premier ordre. Ses danses évoquent distinctement la statuaire indienne au travers de gestes et de poses hiératiques. Ce lien avec la sculpture se manifeste également dans ses travaux plastiques, sous la forme de nombreux photomontages dans lesquels elle substituait des clichés de sa silhouette à des images d'œuvres d'art qu'elle imitait, en conservant le piédestal qui renvoyait à l'œuvre d'art originale $^{44}$ (fig. 5). Jahn souligne le double mécanisme mis en place par la danseuse, à la fois ancré dans la pratique rituelle et tourné vers le progrès scientifique, en combinant l'histoire de l'art et l'image photographique ${ }^{45}$.

L'utilisation de l'art indien par Nyota Inyoka repose sur une étude attestée de l'histoire de l'art mais aussi de l'iconographie, de la philosophie et de la littérature indiennes, fruit de sa rencontre avec de prestigieux savants de l'époque ${ }^{46}$. Cette approche de type transcendantal, bien que partagée par d'autres danseuses, acquiert chez elle une dimension particulièrement 
importante. Son attachement aux sources justifiait qu'on qualifiât ses danses d'évocations et de reconstitutions, tandis que les résonances mystiques de ses pièces incitaient à lui prêter la faculté d'incarner les divinités hindoues. À tout cela se mêlait une revendication, par l'artiste, de l'originalité de ses danses et de ses capacités créatives, qui la menait à une démarche de type intuitif teintée d'ésotérisme ${ }^{47}$. Comme l'écrivait Loulou Roudanez :

La danse égale la sculpture. Il fallait être une très grande danseuse pour toucher aux dieux et à la statuaire antique sans nous décevoir, et avoir une remarquable intuition pour devenir le mouvement et le rythme qui expriment la beauté et l'idée du symbole ${ }^{48}$.

Tórtola Valencia, elle aussi, invoquait sa créativité, assurant composer ses danses à l'intuition - une affirmation que contredisait, comme le fait observer Isabel Clúa, son étude rigoureuse des sources visuelles ${ }^{49}$. La presse attribuait de même une approche intuitive de la danse à Roshanara, formée de surcroît par la jeune Tórtola Valencia juste après que celle-ci eut présenté ses "danses orientales" dans Kismet à Londres ${ }^{50}$. L'historien Coomaraswamy allait quant à lui défendre l'authenticité de la danseuse, invoquant sa formation ultérieure auprès de sa deuxième épouse, Ratan Devi, de son vrai nom Alice ${ }^{51}$.

Nyota Inyoka entoura son labeur créatif d'un halo mystique, voire ésotérique, décrivant les visions de gestes, d'attitudes et de divinités qui lui parvenaient dans l'état de rêverie qui $s^{\prime}$ imposait à elle comme une révélation pendant son travail de composition ${ }^{52}$. L'évocation constante, par l'artiste, de ses visions et de son approche intuitive de la danse, tout comme la revendication de ses origines indiennes, eurent pour conséquence d'occulter son travail créatif dans le sillage tardif de l'orientalisme, un sort qu'elle partagea avec d'autres danseuses de l'époque. Cela leur valut à presque toutes d'être oubliées, l'historiographie des débuts de la danse moderne les ayant jusque très récemment ignorées. Les inspirations orientalistes de ces danseuses allaient les cantonner dans des domaines où la dimension créative de leurs compositions serait éclipsée. D'un côté, elles-mêmes n'eurent de cesse de revendiquer des origines asiatiques plus ou moins fantasmées et de défendre le caractère authentique de leurs danses, issues de traditions " ancestrales ", réduisant d'autant leur potentiel créatif. De l'autre, comme nous l'avons montré, beaucoup d'entre elles prétendaient que leurs danses leur avaient été révélées dans un état de rêverie, d'hypnose ou d'inconscience, s'érigeant en quelque sorte en médiums dont les mouvements auraient été dictés par un agent extérieur. Elles redevenaient ainsi des sujets passifs de leurs propres danses. Cette image, en plus de trouver un écho dans les mouvances spirites et ésotériques si présentes dans les cénacles culturels européens du début du $\mathrm{XX}^{\mathrm{e}}$ siècle, est aussi conforme au topique de la danseuse indienne forgé au siècle précédent. Les descriptions de danses indiennes exécutées dans un état d'exaltation, de délire, voire d'hystérie sont récurrentes, et finissent par aboutir à l'association entre la danseuse indienne et la femme fatale fin de siècle ${ }^{53}$. Ces stéréotypes, qui sous-tendent les descriptions de danses indiennes figurant dans les récits de voyages et les ouvres littéraires et visuelles des XIX ${ }^{e}$ et $\mathrm{XX}^{\mathrm{e}}$ siècles, et même dans les brochures publicitaires des danseuses hindoues, concourent à présenter ces femmes comme des sujets passifs, exécutant des danses dans un état d'inconscience. Tout cela, combiné à l'image orientaliste héritée du romantisme, à laquelle renvoient les danses hindoues et qui évoque invariablement d'anciennes pratiques sacrées, contribuerait à ancrer leur travail dans un exotisme atemporel et transrégional, a priori très éloigné du monde de la danse moderne dans lequel elles développèrent réellement leur travail.

Cette inspiration largement puisée aux sources visuelles donna lieu à des pièces d'un hiératisme marqué, également lié à leur caractère sacré, et fortement imprégnées de la gestuelle si codifiée de l'art et de la danse indiennes. Ainsi se mettait en place un jeu suggestif entre la rigidité de la sculpture et le mouvement inhérent à la danse, articulé 
par le corps de la danseuse. L'évocation d'œuvres statiques par les danseuses hindoues correspondait à la perception générale des danses indiennes ; cette capacité de donner vie à des images pétrifiées allait devenir récurrente. On la retrouve chez Rodin s'exclamant, à propos des danseuses cambodgiennes, "les frises d'Angkor s'animaient sous mes yeux $^{54}$ ". Sans cesser de les regarder, il se hâta de reproduire leurs gestes et attitudes sur le papier afin de les capturer de nouveau ${ }^{55}$. Francis de Miomandre affirmait que Nyota Inyoka était capable d'insuffler la vie à ses sources, et mettait en avant le rôle du corps pour incarner l'imaginaire évoqué dans ses danses :

Tout ce que l'Orient est pour nous, tout ce qu'il nous apporte de grave, de religieux, de pur, ce consentement aux yeux fermés, ces gestes d'offrande et de propitiation, cet immense abandon aux forces éternelles, Nyota Inyoka l'incarne pour nous [...]. Qu'elle a beaucoup étudié les fresques et les bas-reliefs, eh ! Barbeau, je le sais bien. Mais il fallait galvaniser cette immobilité, ces gestes pétrifiés par l'art plastique, au point que nous les croyions des attitudes à jamais fixes de la prière ou l'extase, il fallait les relier entre eux, deviner leurs transitions, reconstruire avec toute cette science léthargique la joie, le mouvement, l'illumination de la vie ${ }^{56}$.

Ces quelques mots synthétisent bien l'entre-deux où se situe la méthode de composition des danses indiennes présentées sur les scènes occidentales dans les premières décennies du XXe siècle. Fondées sur l'étude des images, des œuvres d'art et de l'ensemble des manifestations culturelles des traditions invoquées, et revendiquant par ailleurs une authenticité plus ou moins légendaire au travers de la construction identitaire à laquelle se livrèrent plusieurs de leurs interprètes, ces pièces s'inscrivent aussi dans les mouvements de la modernité et de l'avant-garde de la première moitié du XX $\mathrm{X}^{\mathrm{e}}$ siècle. Francis de Miomandre évoquait ainsi la créativité de la danseuse pour traduire ses sources visuelles en un mouvement capable de susciter la danse et, en définitive, de donner vie à ces œuvres statiques. Selon un poncif courant dans l'histoire de l'art, la danse incarne alors, depuis sa corporalité intrinsèque, de nombreux enjeux de la modernité. Rien d'étonnant, donc, à ce que la danse moderne occupe une place fondamentale sur la scène artistique de l'époque.

Cette conception vitale de l'art, qui rapproche la danse d'autres formes d'expression, s'articule autour de la notion de rythme en tant que pulsion interne de l'œuvre ${ }^{57}$, dont l'analogie avec la notion indienne de chanda, interprétée par Abanindranath Tagore dans son Șadanga, n'est sans doute pas anodine ${ }^{58}$. "Chanda signifie : ce qui donne l'élévation, ce qui oblige à se mouvoir harmonieusement dans une joyeuse exaltation ", écrit l'artiste bengali, qui définit cette pulsion comme « le mouvement de la vie ». Ce rythme, qui semble imprimer un mouvement vital à l'œuvre d'art, est symbolisé dans le Națarāja par le damaru (tambour) que porte Shiva dans sa main supérieure droite, interprété à son tour par Coomaraswamy comme le rythme originel du monde qui met en mouvement, en même temps que la danse du dieu, l'ensemble des particules de l'univers ${ }^{59}$ (fig. 6).

Ce commentaire du Natarāja, alors très connu en Inde et en Occident, est une référence incontestable pour Nyota Inyoka et Uday Shankar. Il véhicule en outre une

6. Victor Goloubew, photographie de Națarāja, XII siècle, Chennai, Government Museum, publiée dans Coomaraswamy, 1922, cité n. 27.

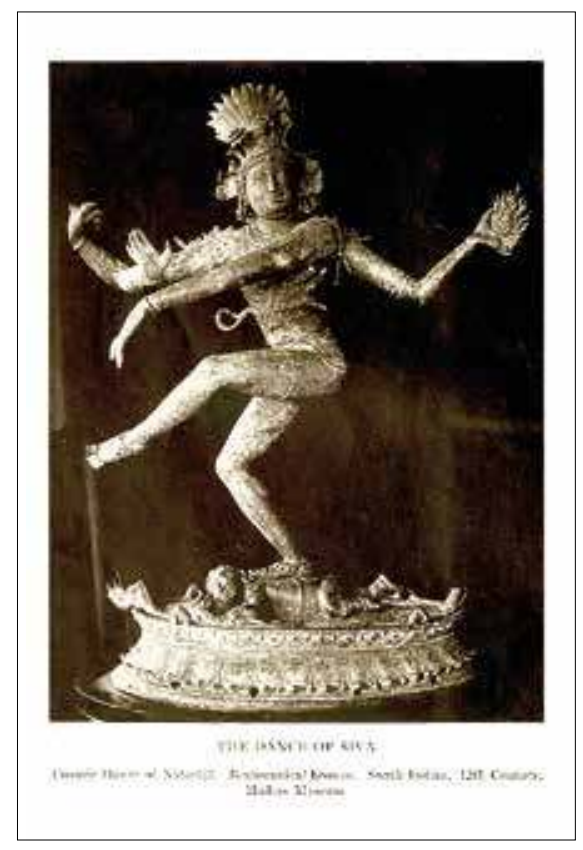


conception cosmique de l'art qui identifie la danse au mouvement des astres lui-même, nous renvoyant d'une part aux diverses civilisations antiques pour qui cette forme d'expression était intimement liée au rituel et au divin, mais aussi, d'autre part, à une modernité qui associait le rythme à l'énergie vitale de l'art. Fernand Divoire, écrivain, occultiste et défenseur de la danse moderne, exprimait ainsi cette analogie entre la danse et le rythme vital inhérent à toute discipline artistique :

N'avons-nous pas vu ainsi que la danse manifeste les rythmes propres à l'architecture, par ses volumes; à la sculpture, par ses équilibres de gestes ; à la musique, par son mouvement ? Et elle vit ! Et, dans une certaine mesure, elle écoute les rythmes du cœur et des poumons, du sang et du souffle ${ }^{60}$.

Ainsi, le rapprochement continuel des danses hindoues avec l'antiquité et le sacré s'articule en un langage qui est aussi celui de la modernité elle-même, dont les liens avec l'ésotérisme sont également avérés. L'idée de l'imbrication des mouvements ésotériques et de l'art moderne est défendue depuis quelques dizaines d'années ${ }^{61}$. Partha Mitter y fait allusion dans son étude sur la réception de l'art indien en Europe, en montrant les liens qu'entretenaient des artistes d'avant-garde comme Piet Mondrian ou Vassily Kandinsky avec la théosophie, dont la relation avec les religions du sous-continent indien ne fait aucun doute ${ }^{62}$. Cependant, des études plus approfondies mettent au jour l'influence, encore trop souvent ignorée, des mouvements ésotériques sur l'art moderne, que ce soit dans le champ de l'abstraction ou dans d'autres formes d'expression plastique. Dans le même ordre d'idées, un ouvrage récent traite de l'influence de l'Inde dans l'art moderne où, comme le soulignait Mitter, la théosophie occupe une place essentielle ${ }^{63}$. Les études consacrées aux liens entre la danse du début du $\mathrm{XX}^{\mathrm{e}}$ siècle et les cercles ésotériques sont encore assez rares, même si de plus en plus de publications y font explicitement allusion ${ }^{64}$.

Le panorama interdisciplinaire de l'art occidental moderne est donc traversé par des questions relatives à une réappropriation de l'Antiquité préindustrielle, liées à leur tour aux inclinaisons de l'ésotérisme occidental moderne pour ce qu'on appelle les " spiritualités alternatives ». On pourrait ajouter à cela un intérêt marqué pour la corporalité et la vitalité de l'art, souvent associée à la danse, qui à la même époque cherche sa place dans les sphères du "grand art " ou de la haute culture. C'est dans ce contexte que s'inscrivent les propositions créatives des danses hindoues qui, au cours des premières décennies du XXe siècle, eurent à cœur d'incarner ces questions, auxquelles s'intéressèrent des artistes comme Auguste Rodin. Les danseuses hindoues eurent également recours dans leur processus de création, aussi complexe qu'obscur, aux sources visuelles et artistiques des cultures qu'elles mettaient en scène, orchestrant de ce fait un ballet de disciplines proche des postulats de nombreux mouvements d'avant-garde. Devant l'importance de réhabiliter à la fois le travail créatif de ces artistes et leur place dans le paysage de la danse moderne, il nous semble nécessaire de repenser les dynamiques qui sous-tendirent l'évolution de la modernité au tournant des XIX et XX ${ }^{\mathrm{e}}$ siècles. À cet égard, les échanges culturels et commerciaux nés de l'impérialisme permettent de révéler de manière éclatante le rôle des cultures non européennes dans la configuration d'une modernité qu'il convient d'appréhender sous l'angle de dynamiques plurielles - une question qui transparaît avec encore plus de force dans les corps alternatifs et subversifs des danseuses hindoues, incarnations à la fois de la modernité et du " caractère ancestral " auquel avait été associé l'imaginaire de l'exotisme.

Cette contribution a été traduite de l'espagnol par Sophie Zambrano. 


\section{Irene López Arnaiz}

Irene López Arnaiz est chercheuse au sein du programme "Juan de la Cierva - Formación » du Consejo Superior de Investigaciones Científicas (CSIC, Madrid). Elle est titulaire d'un doctorat en histoire de l'art de l'Universidad Complutense de Madrid ("cum laude", mention internationale, 2018). Entre 2014 et 2018, elle a été rattachée à I'Universidad Complutense dans le cadre d'un contrat prédoctoral et a effectué des séjours de recherche à Paris (INHA et CEIAS) et à Londres (V\&A Museum).

\section{NOTES}

Cette recherche a pu être menée grâce à un contrat pré-doctoral du ministère de l'Économie et de la Compétitivité du Gouvernement espagnol (BES-2013062622) et s'inscrit dans les projets de recherche "Trama " (HAR2017-82394-R) et "Sílfide " (PGC2018093710-A-I00), financés par l'AEI / MCI [Agence gouvernementale de la recherche- ministère de la Science et de I'Innovation] et le FEDER (UE). Les révisions du texte ont été effectuées dans le cadre d'un contrat postdoctoral "Juan de la Cierva - Formación » (FJC2018035818-I) du ministère de la Science et de I'Innovation du Gouvernement espagnol.

1. "La sensualità ha raggiunto soprattutto in Oriente, espressione singolare d'una sorta di misticismo carnale di cui vedemmo esempi in riti greci e romani e in certe strane feste medioevali da noi. " Anton Giulio Bragaglia, Scultura vivente, Milan, L'Eroica, 1928, p. 16. C'est nous qui traduisons, $\mathrm{NdT}$.

2. Décoret-Ahiha a ouvert un champ d'études fondamental, qui ne cesse de s'accroître: Anne Décoret-Ahiha, Les danses exotiques en France, 1900-1940, 5 vol., thèse Université Paris VIII, 1998 ; Anne Décoret-Ahiha, Les danses exotiques en France (1880-1940), Pantin, Centre national de la danse, 2004.

3. Un panorama de cette question a été présenté avec d'autres formes scéniques par Décoret-Ahiha, 1998, cité n. 2 ; Décoret-Ahiha, 2004, cité n. 2 et Claudia Palazzolo, Mise en scène de la danse aux expositions de Paris - 1889-1937. Une fabrique du regard, Paris, L'CEil d'or, 2017. D'autres études ont porté sur les danses d'inspiration indienne créées en Occident en rapport avec les processus de reconfiguration de la danse classique indienne : Tiziana Leucci, Devadāsī e Bayadères: tra storia e leggenda. Le danzatrici indiane nei racconti di viaggio e nell'immaginario teatrale occidentale (XIIIXX secolo), Bologne, CLUEB, 2005 ; Tiziana Leucci, Du Dâsî Âttam, au Bhârata Nâtyam: ethno-histoire d'une tradition chorégraphique et de sa moralisation \& nationalisation dans I'Inde coloniale et post-coloniale, vol. 1, thèse, Paris, École des hautes études en sciences sociales; Ananda Ceballos, La danza Odiśí como construcción de un clasicismo regional. Estudio estético de una tradición coreográfica de la India contemporánea, thèse, Saint-Sébastien, Universidad del País Vasco, 2013. On trouvera par ailleurs une analyse transdisciplinaire des danses indiennes et des arts visuels dans le contexte français dans la thèse doctorale : Irene López Arnaiz, La impronta de las danzas " indias » en Francia. El encuentro de las artes plásticas y escénicas, entre el exotismo y la modernidad (18381939), Madrid, Universidad Complutense, 2018. On citera également de précieuses études menées récemment qui redonnent une place d'importance aux compositions d'inspiration orientaliste sur la scène occidentale dans les prémices de la danse moderne : Ramsay Burt, Michael Huxley, Dance, Modernism, and Modernity, Londres / New York, Routledge, 2020 ; Edward Ross Dickinson, Dancing in the Blood. Modern Dance and European Culture on the Eve of the First World War, Cambridge, Cambridge University Press, 2017. II faudrait ajouter à tout cela plusieurs monographies consacrées aux danseurs et danseuses de l'époque qui pratiquèrent les danses indiennes, dont les références sont citées ponctuellement tout au long du présent essai. Toutefois, comme nous le soulignons un peu plus loin, notre étude s'intéresse à un aspect qui nous est apparu comme essentiel dans le processus créatif de ces artistes qui, en se tournant délibérément vers des traditions ancestrales, se livrèrent à un usage et une étude remarquables des sources visuelles asiatiques, s'inscrivant du même coup dans le mouvement de la modernité.

4. André Levinson, La danse d'aujourd'hui, Paris, Éditions Duchartre, 1929, p. 282.

5. Théophile Gautier, Voyage en Espagne (Tra los montes), Paris, G. Charpentier et Cie, 1842, p. 31. Ángel González García cite ce passage dans son essai éloquent sur la réception du « caractère espagnol » par l'avant-garde internationale. Ángel González García, "La noche Española », dans El Resto. Una historia invisible del arte contemporáneo, Madrid, Museo de Bellas Artes de Bilbao / Museo Centro de Arte Reina Sofía, 2000, p. 84-95, notes des p. 484-488.

6. Henry Fouquier, "Autour de l'Exposition », dans Émile Monod, L'Exposition Universelle de 1889. Grand ouvrage illustré, historique, encyclopédique, descriptif, Paris, É. Dentu Éditeur, 1890, vol. 1, p. 280.

7. Arthur Pougin, Le Théâtre à l'Exposition universelle de 1889, Paris, Librairie Fischbacher, 1890, p. 120.

8. Palazzolo, 2017, cité n. 3.

9. Voir Joep Bor, « Mamia, Ammani and other Bayadères: Europe's Portrayal of India's Temple Dancers ", dans Martin Clayton, Zon Bennet (dir.), Music and Orientalism in the British Empire, 1780-1940's. Portrayal of the East, Hampshire / Burlington, Ashgate, 2007, p. 39-70 ; Leucci, 2005, cité n. 3 ; Tiziana Leucci, «Fascinantes bayadères, mais étrange musique... Réception française des danseuses indiennes : des récits de voyage aux œuvres de É. de Jouy, T. Gautier et H. Berlioz ", dans Luc Charles-Dominique, Yves Defrance, Danièle Pistone (dir.), Fascinantes étrangetés. La découverte de l'altérité musicale en Europe au XIXe siècle, Paris, L'Harmattan, 2014, p. 343-365.

10. Catherine Servan-Schreiber, « Les spectacles de danses indiennes des Expositions Coloniales et Universelles, 1878-1934 », dans Tiziana Leucci, Raphaël Rousseleau, 
De la "danse de Shiva " à la World Music. Icône du rythme et langage des gestes entre I'Inde, l'Europe et les États-Unis (à paraître) ; Décoret-Ahiha, 1998, cité n. 3.

11. Palazzolo, 2017, cité n. 3, p. 40-52 et 141-167 ; López Arnaiz, 2018, cité n. 3, p. 193-229; 276-295 et 471-502.

12. Aurélie Samuel (dir.), Du Nô à Mata Hari. 2000 ans de théâtre en Asie, cat. exp. (Paris, musée Guimet), Paris, Éditions Artlys, 2015, p. 135-138; Mathew Isaac Cohen, Performing Otherness. Java and Bali on International Stages, 1905-1952, Hampshire / New York, Palgrave Macmillan, 2010, p. 23-35 ; Catherine Authier, Femmes d'exception, femmes d'influence. Une histoire des courtisanes au XIXe siècle, Malakoff, Armand Colin, 2015, p. 255-260.

13. Cléo de Mérode, Le ballet de ma vie (1955), Paris, Pierre Horay, 1985, p. 222-230.

14. Décoret-Ahiha, 1998, cité n. 3, p. 248 et Annexe 3, Cosika Vrondja, s.p. ; Palazzolo, 2017, cité n. 3, p. 169248

15. Programme Anna Pavlova, Royal Opera House Covent Garden, 8 septembre -4 octobre 1924. Ceballos, 2013, cité n. 3, p. 109.

16. Joan L. Erdman, "Performance as a Translation. Uday Shankar in the West ", dans The Drama Review, vol. $31, n^{\circ} 1,1987$, p. 65-88.

17. Voir Diana Brenscheidt, Shiva onstage. Uday Shankar's Company of Hindu Dancers and Musicians in Europe and the United States, 1931-1938, Zürich, Berlin, LIT Verlag, 2011 ; Ruth K. Abrahams, "Uday Shankar: The Early Years, 1900-1938 ", dans Dance Chronicle, vol. 30, 2007, p. 363-426.

18. Palazzolo, 2017, cité n. 3, p. 50.

19. «La Nuit du Grand Palais » dans L'Illustration, 27 juin 1925 ; programme Nyota Inuoka dans ses danses sacrées de l'Inde et de l'Égypte, Théâtre de l'Exposition des arts décoratifs, Paris, 30 mai-1 ${ }^{\text {er }}$ juin 1925.

20. Pierre Thiriot, "In Memoriam. Jeanne Ronsay ", dans Toute la danse, $\mathrm{n}^{\circ}$ 12, juin 1953 ; Charlotte Arrighi De Casanova (dir.), Simkie, Paris-Delhi, Mareterraniu, 2009 (documentaire).

21. "A global dimension of modernism » - c'est nous qui traduisons, $\mathrm{NdT}$.

22. Burt et Huxley, 2020, cité n. 3, p. 5.

23. Programmes Le Monde Colonial qui chante et qui danse, Exposition coloniale de 1931, Paris, 1931 ; Uday Shan-Kar, Comédie des Champs-Élysées, Paris, 14-20 juin 1937 ; Djemil-Anik avec Raden Mas Waloejo et sa partenaire Retna Moerindiah, Théâtre des ChampsÉlysées, 7 juillet 1937 ; Danses de l'Inde et de l'Égypte par Nyota Inyoka. Danseuses de I'Inde, Théâtre des Champs-Élysées, 29 septembre 1937. Fernand Divoire, "La danse », dans La Revue de France, 11 décembre 1931. Voir Jacqueline Robinson, L'aventure de la danse moderne en France (1920-1970), Clamecy, Éditions Bougé, p. 87-96.

24. Gayatri Chakravorty Spivak, Can the subaltern speak?, Basingstoke, Macmillan, 1988 ; Homi K. Bhabha, The Location of Culture, Londres / New York, Routledge, 1994 ; Édouard Glissant, Traité du tout-monde, Paris, Gallimard, 1997 ; Partha Mitter, « Decentering
Modernism: Art History and Avant-Garde Art from the Periphery ", dans The Art Bulletin, vol. 90, n 4, décembre 2008, p. 531-548; Walter Mignolo et Catherine W. Walsh, On Decoloniality: Concepts, Analytics and Praxis, Durham, Duke University Press, 2018.

25. Francis de Miomandre, Danse, Paris, Flammarion, 1935 , p. 3

26. Voir Dickinson, 2017, cité n. 3, p. 27. Les processus de légitimation de la danse qui caractérisent les débuts de la danse moderne, moyennant l'intégration de celle-ci aux contextes artistiques et culturels de l'époque, ainsi que la remise en question de la classification entre espaces officiels et alternatifs, ont été étudiés à partir des créations de nombreux danseurs de la période. La présente étude reprend les lignes proposées, notamment, dans les travaux suivants : Dickinson, 2017, cité n. 3 ; Cohen, 2010, cité n. 12 ; Iris Garland, Tórtola Valencia. Modernism and Exoticism in Early Twentieth Century Dance, Vancouver, FIVE / CINQ, 2013 ; Idoia Murga Castro (dir.), Poetas del cuerpo. La danza de le Edad de Plata, cat. exp. (Madrid, Residencia de Estudiantes), Madrid, Publicaciones Residencia de Estudiantes, 2017.

27. Par exemple : Ernest Binfield Havell, Indian Sculpture and Painting Illustrated by Typical Masterpieces, with an Explanation of their Motives and Ideals, Londres, John Murray, 1908 ; Sylvain Lévi, Le théâtre indien, Paris, É. Bouillon, 1890 ; Ananda Coomaraswamy (éd.), The Mirror of Gesture. Being the Abhinaya Darpana of Nandikeśvara, Ananda Coomaraswamy et Gopala Kristnayya Duggirala (trad. angl.), Cambridge, Harvard University Press, 1917 ; Ananda Coomaraswamy, La Danse de Çiva. Quatorze essais sur I'Inde (1918), Madeleine Rolland (trad. fr.), Romain Rolland (avant-propos), Paris; George Groslier, Danseuses cambodgiennes, anciennes et modernes, Paris, Augustin Challamel, 1913.

28. Voir Pierre Baptiste et Thierry Zéphir (éd.), Angkor Naissance d'un mythe. Louis Delaporte et le Cambodge, cat. exp. (Paris, Musée national des arts asiatiquesGuimet, 2013-2014), s.l., Gallimard / Musée national des arts asiatiques-Guimet, 2013 ; Marco Deyasi, French Visual Culture and "Indochina »: Modernism, Primitivism, and Colonialism, 1889-1931, thèse, Durham, Duke University, 2007.

29. Anonyme [Nyota Inyoka], L'Art de Nyota Inyoka, tapuscrit inédit, musée des Années Trente.

30. Voir Gabriele Brandstetter, Poetics of Dance. Body, Image, and Space in the Historical Avant-Gardes (Francfort, 1995), New York, Oxford University Press, 2015, p. 25-88.

31. Mérode, 1955, cité n. 13, p. 223. La danseuse s'inspirait aussi des images en mouvement qu'offrait le cinéma naissant.

32. Identifié avec un bronze des XVIII ${ }^{e}-X I X{ }^{e}$ siècles (MG18529). On aperçoit au fond de la scène une statue en granit provenant du Tamil Nadu qui représente le dieu Skanda, datée des XIVe-XVe siècles (MG18528).

33. Voir Brandstetter, 2015, cité n. 30, p. 66-68; Romita Ray, "Orientalizing the Bayadère / Fabricating Mata Hari ", dans Photographies, vol. 5, n 1, 2012, p. 87-111; Alexandra Kolb, «Mata Hari's in the Context of Femininity 
and Exoticism », dans Mandrágora, no 15, 2009, p. 58-79 ; Leucci, 2009, cité n. 3, vol. 1, p. 152-154.

34. Ruth Saint Denis, An Unfinished Life (vers 1939), Londres / Mumbai / Sidney, George G. Harrap \& Company, p. 55.

35. Leucci, 2005, cité n. 3, p. 239.

36. " [L] egittimazione della propia ricerca religiosa » - c'est nous qui traduisons, NdT.

37. Voir Susan Tenneriello, "The Divine Spaces of Metaphysical Spectacle: Ruth St Denis and Denishawn Dance Theatre at Lewisohn Stadium, the esoteric model in American performance ", dans Performance Research, vol. 13, nº 3, 2009, p. 124-138 ; Uttara Asha Coorlawala, "Ruth Saint Denis and India », dans Dance Chronicle, vol. 15, n 2, 1992, p. 123-152 ; Jane Desmond, « Dancing Out the Difference: Cultural Imperialism and Ruth St. Denis's Radha of 1906 », dans Signs, vol. 17, n 1, printemps 1991, p. 28-49.

38. Saint Denis, vers 1939, cité n. 34, p. 70.

39. John Ernest Crawford Flitch, Modern Dancing and Dancers, Londres, Grant, Ritchards LTD, Joshua B. Lippincott Company, 1912, p. 191-194.

40. Voir Carmen de Burgos Segui (Colombine), Confidencias de artistas, Madrid, Sociedad Española de Librería, 1917, p. 185-186 ; Michelle Clayton, « Touring History: Tórtola Valencia Between Europe and the Americas ", dans Dance Research Journal, vol. 44, nº 1, été 2012, p. 34 ; Garland, 2013, cité n. 27, p. 45.

41. Garland, 2013, cité n. 27, p. 75.

42. André Levinson, "La danse. Miniatures et bibelots", dans Candide, s.d., Paris, BNF, fonds Nyota Inyoka.

43. Lugné-Poe, "La danseuse Nyota Inyoka. Évocatrice de l'Orient ", dans La Danse, novembre 1921.

44. Le Fonds Nyota Inyoka de la BNF conserve encore ces montages inédits montrant la danseuse dans des poses et des attitudes imitées de sculptures et de miniatures égyptiennes, indiennes, ceylanaises, cambodgiennes et indonésiennes. Trois de ces montages furent reproduits dans le programme Nyota Inyoka au bénéfice de l'œuvre "Le colis tricolore ", Salle Gaveau, Paris, 25 avril (BMO). La sculpture du montage présenté ici a été identifiée avec un bodhisattva reproduit par Coomaraswamy. Voir par exemple Ananda K. Coomaraswamy, The Arts and Crafts of India \& Ceylon, New Delhi, Today \& Tomorrow's Publishers, 1913, p. 61 ; Ananda K. Coomaraswamy, Visvakarma: examples of Indian architecture, sculpture, painting, handicraft, Londres, Messrs. Luzac, 1914, L. 13-14.

45. Voir Tessa Jahn, "Cutting into History: the Hindu Dancer Nyota Inyoka's photomontages », dans Christoph Wulf (dir.), Exploring Alterity in a Globalized World, Londres / New York, Routledge, 2016, p. 187-196.

46. Voir Irene López Arnaiz, "Movimientos imaginados, gestos rastreados. Nyota Inyoka, una (re)creación mestiza de las danzas indias », dans Eva Fernández del Campo, Sergio Román Aliste (dir.), Las mujeres que inventaron el arte indio, Madrid, Asimétricas (à paraître).

47. Levinson, s.d., cité n. 42 ; Jean Rollot, "La danse hindoue aux Variétés », dans Ce matin, 19 avril 1932.
48. Loulou Roudanez, Des danseurs qui sortent du cadre. Nyota Inyoka, Paris, J. Susse, 1947, p. 54.

49. Isabel Clúa, Cuerpos de escándalo. Celebridad femenina en el fin-de-siècle, Barcelone, Icaria, 2016, p. 132.

50. Anonyme, "An Indian taught by a Spanish dancer: Mlle Roshanara ", dans The Sketch, 12 juillet 1911, p. 9.

51. Ananda K. Coomaraswamy, "Oriental Dances in America. And a Word or Two in Explanation of the Nautch », dans Vanity Fair, mai 1912.

52. Roudanez, 1947, cité n. 48, p. 73-74.

53. On citera notamment les intéressantes descriptions de Louis Jacolliot et de Pierre Loti : Louis Jacolliot, Voyage au Pays des Bayadères. Les mœurs et les femmes de I'Extrême-Orient, Paris, Albert Lacroix, 1875, p. 263279 ; Pierre Loti, L'Inde sans les Anglais, Paris, CalmannLévy, 1903, p. 217-222. Voir Ceballos, 2013, cité n. 3, p. 39-40 et López Arnaiz, 2018, cité n. 3, p. 122-131 et 359-372.

54. Auguste Rodin, cité dans L'Art moderne, 30 juin 1912.

55. Voir Dominique Viéville, Jacques Vilain (dir.), Rodin et les danseuses cambodgiennes. Sa dernière passion, cat. exp. (Paris, musée Rodin), Paris, Éditions du musée Rodin, 2006 ; Juliet Bellow, " Hand Dance: Auguste Rodin's Drawings of the Cambodian Royal Ballet», dans The Art Bulletin, vol. 101, n 3, 2019, p. 37-65.

56. Francis de Miomandre, La Semaine, 7 janvier 1933, cité dans le programme Nytota Inyoka et ses ballets (s.d) (BNF, Ms-13783-84).

57. Voir Teresa Aizpún, Cayetana Ibáñez et Eva Fernández del Campo (dir.), Ritmo. El pulso del arte y de la vida, Madrid, Abada, 2015.

58. Abanindranath Tagore, Sadanga ou les six Canons de la Peinture hindoue (trad. Andrée Karpèles), Paris, Éditions de Bossard, 1922, p. 38-39. Ce texte est d'abord paru en 1914 en bengali et en anglais. Voir Sergio Román Aliste, Dimensiones artísticas de la pedagogía en Śāntiniketan: un proyecto internacional en el medio rural bengalí (1901-1951), thèse, Madrid, Universidad Complutense, p. 412-423.

59. Coomaraswamy, 1918, cité n. 27, p. 56-66.

60. Fernand Divoire, "Le Rythme dans tous les Arts » (conférence des 9 et 11 février 1929), dans Conferencia, journal de l'Université des Annales, s.d. (BNF).

61. Bien que cette question soit abordée dans plusieurs monographies, nous citons ci-après les principales études générales. La première exposition à mettre l'accent sur ce thème fut Maurice Tuchman (dir.), The Spiritual in Art: Abstract Painting 1890-1895, cat. exp. (Los Angeles, Los Angeles County Museum / Chicago, Museum of Contemporary Art / La Haye, Haags Gemeentemuseum, 1986-1987), New York, Abbeville Publishers, 1987. Elle fut suivie des expositions Alfred Pacquement, Jean de Loisy et Angela Lampe (dir.) Traces du sacré, cat. exp. (Paris, Centre Pompidou, 2008), Paris, Centre Pompidou, 2008 ; Serge Fauchereau et Joëlle Pijaudier-Cabot (dir.), L'Europe des esprits ou la fascination de l'occulte, 1750-1950, cat. exp. (Strasbourg, musée d'Art moderne et contemporain / Berne, Zentrum Paul Klee, 2011-2012), Strasbourg, Musées de Strasbourg, 2011 ; Francesco Parisi (dir.), Arte e magia. Il fascino 
dell'esoterismo in Europa, cat. exp. (Rovigo, Palazzo Roverella, 2018-2019), Milan, Silvana Editoriale, 2018. Les écrits de Marco Pasi font autorité dans ce champ d'études, qu'il s'agisse d'arts visuels ou de littérature: Marco Pasi, " Hilma af Klint, el esoterismo occidental y el problema de la creatividad artística moderna », dans Boletín de Arte, n³ 35, 2014, p. 43-59; Marco Pasi, "Varieties of Magical Experience: Aleister Crowley's View on Occult Practice ", dans Magic, Ritual, and Witchcraft, hiver 2011, p. 123-162. Dans le domaine académique espagnol, on citera la publication d'Alberto Luque, Arte moderno y esoterismo, Lleida, Editorial Milenio, 2002.

62. Partha Mitter, Much Maligned Monsters. A History of European Reactions to Indian Art, Chicago / Londres, The University of Chicago Press, p. 274. II développera cet aspect dans Mitter, décembre 2008, cité n. 14, p. 537-538. En outre, la thèse de Deyasi sur l'influence de l'Indochine dans I'art français de la fin du XIXe et du début du XXe siècle fait une large part à l'ésotérisme.

63. Naman P. Ahuja, Louise Belfrage (dir.), A Mediated Magic. The Indian Presence in Modernism, 1880-1930, Mumbai, Marg, 2019.

64. Dickinson, 2017, cité n. 3, p. 166-159; Burt et Huxley, 2020, cité n. 3, p. 73-93; Adrien Sina (dir.), Feminine Futures. Valentine de Saint-Point. Performance, Danse, Guerre, Politique et Érotisme, Paris, Les Presses du réel, 2011 ; López Arnaiz, à paraître, cité n. 46. 